\title{
Utilization Patterns of Oral Disease-Modifying Drugs in Commercially Insured Patients with Multiple Sclerosis
}

\author{
Rishi J. Desai, MS, PhD; Mufaddal Mahesri, MD, MPH; Joshua J. Gagne, PharmD, ScD; \\ Eimir Hurley, MS; Angela Tong, MS; Tanuja Chitnis, MD; Sarah Minden, MD; Claire M. Spettell, PhD; \\ Olga S. Matlin, PhD; William H. Shrank, MD, MS; and Niteesh K. Choudhry, MD, PhD
}

\begin{abstract}
BACKGROUND: The approval of new oral disease-modifying drugs (DMDs), such as fingolimod, dimethyl fumarate (DMF), and teriflunamide, has considerably expanded treatment options for relapsing forms of multiple sclerosis (MS). However, data describing the use of these agents in routine clinical practice are limited.
\end{abstract}

OBJECTIVE: To describe time trends and identify factors associated with oral DMD treatment initiation and switching among individuals with MS.

METHODS: Using data from a large sample of commercially insured patients, we evaluated changes over time in the proportion of MS patients who initiated treatment with an oral DMD and who switched from an injectable DMD to an oral DMD between 2009 and 2014 in the United States. We evaluated predictors of oral DMD use using conditional logistic regression in 2 groups matched on calendar time: oral DMD initiators matched to injectable DMDs initiators and oral DMD switchers matched to those who switched to a second injectable DMD.

RESULTS: Our cohort included 7,576 individuals who initiated a DMD and 1,342 who switched DMDs, of which oral DMDs accounted for $6 \%$ and $39 \%$, respectively. Oral DMD initiation and switching steadily increased from $5 \%$ to $16 \%$ and $35 \%$ to $84 \%$, respectively, between 2011 and 2014 , with DMF being the most commonly used agent. Of the potential predictors with clinical significance, a recent neurologist consultation $(O R=1.60 ; 95 \%$ $\mathrm{Cl}=1.20-2.15)$ and emergency department visit $(\mathrm{OR}=1.43 ; 95 \% \mathrm{Cl}=1.01$ 2.01) were significantly associated with oral DMD initiation. History of depression was noted to be a potential predictor of oral DMD initiation; however, the estimate for this predictor did not reach statistical significance $(\mathrm{OR}=1.35 ; 95 \% \mathrm{Cl}=0.99-1.84)$. No clinically relevant factors measured in our data were associated with switching to an oral DMD.

CONCLUSIONS: Oral DMDs were found to be routinely used as second-line treatment. However, we identified few factors predictive of oral DMD initiation or switching, which implies that their selection is driven by patient and/or physician preferences.

J Manag Care Spec Pharm. 2019;25(1):113-21

Copyright @2019, Academy of Managed Care Pharmacy. All rights reserved.

\section{What is already known about this subject}

Injectable disease-modifying drugs (DMDs) have been a cornerstone of care for relapsing-remitting forms of multiple sclerosis (MS), but nonadherence to these agents is known to be high due, in part, to the need for carefully maintaining self-injection schedules and various adverse effects, such as injection site reactions. The approval of the oral DMDs fingolimod, teriflunamide, and dimethyl fumarate as alternatives to injectable agents has been met with enthusiasm.

\section{What this study adds}

This study demonstrated widespread adoption of oral DMDs in routine clinical practice as alternatives to injectable DMDs for MS, especially for second-line treatment; use of oral DMDs as first-line treatment options for MS was uncommon.

Oral agents accounted for the majority of treatment switches, suggesting that providers may be prescribing these agents in situations of failure of the platform injectable agents or strong patient and physician preferences.

A high frequency of switching to oral DMDs highlights the need for continued research on optimal switching approaches to ensure maximum benefits among patients.

T njectable disease-modifying drugs (DMDs), including interferon-beta formulations and glatiramer acetate, have been L a cornerstone of care for relapsing-remitting forms of multiple sclerosis (MS), since they can reduce rates of relapse and improve quality of life. ${ }^{1}$ However, nonadherence to these agents is known to be high, reported in $13 \%-46 \%$ of patients, ${ }^{2}$ due, in part, to the need for carefully maintaining self-injection schedules and various adverse effects, such as injection site reactions. It is, therefore, unsurprising that the approval of the oral DMDs fingolimod, teriflunamide, and dimethyl fumarate (DMF) as alternatives to injectable agents has been met with enthusiasm. Higher adherence with oral DMDs compared with injectable DMDs has been reported in routine care patient populations. ${ }^{3}$

Unfortunately, oral DMDs are not without potential downsides. While data from the last 2 decades of clinical experience demonstrate the relatively low risk of serious adverse events such as infections associated with the first-line 
(platform) injectable DMDs, ${ }^{4}$ the more potent immunosuppressive actions of the oral agents have come under scrutiny because of their potential association with serious infections, including varicella zoster virus infections and progressive multifocal leukoencephalopathy. ${ }^{5-7}$ Other agent-specific adverse events, including bradycardia with fingolimod, gastrointestinal side effects with DMF, and teratogenicity with teriflunamide, have also been documented. ${ }^{8}$

In the absence of established clinical guidelines by national or international neurology societies regarding selection of DMDs, the decision to use oral DMDs in relapsing-remitting MS is generally thought to be based on patient or provider preferences or a clinical need as demonstrated by inadequate response or intolerability with the platform agents. ${ }^{9}$ However, little is known about the adoption of oral DMDs in routine clinical practice and the factors influencing their use. A better understanding of these issues is critical to inform policymakers and clinical guideline developers. Therefore, using data from a large nationwide commercial insurance program, we designed this study to describe time trends in utilization of oral DMDs in MS and patient factors associated with their use.

\section{Methods}

\section{Data Source and Patient Population}

Health care utilization claims data from Aetna, a large national health insurer in the United States that covers approximately 11 million lives, were used for the period of January 2009-December 2014 to identify a cohort of patients with MS. This data source includes a Health Insurance Portability and Accountability Act (HIPAA)-limited dataset containing patientlevel information on demographic characteristics, outpatient services use, hospitalizations, and dispensed prescriptions, which can be tracked longitudinally. This study was approved by the Institutional Review Board of Brigham and Women's Hospital.

\section{Study Cohort}

We identified patients with a diagnosis of MS (International Classification of Diseases, Ninth Revision, Clinical Modification code 340.xx) recorded on an outpatient or inpatient medical claim. We defined each patient's index date as the date when the first MS diagnosis code appeared on a medical claim during the study period. To ensure the comprehensive availability of health care data, included patients were required to have had continuous insurance enrollment for at least 180 days before this date. We followed these patients for filling of DMD prescriptions beginning on the index date until their health plan disenrollment and included 2 groups of patients in this study: those who initiated treatment after the index date (defined as individuals who had not previously filled any DMD prescriptions) and those who switched from an injectable DMD to either another injectable or an oral alternative after the index date.
For patients with multiple switches after the index date, only the first switch was included. The approach of including patients with a diagnosis code for MS and at least 1 filled DMD prescription has been noted to have a high positive predicted value for MS (0.88-0.92) in administrative claims..$^{10}$ In addition to the 3 oral DMDs (DMF, fingolimod, and teriflunomide), we included the following injectable agents: interferon-beta la, interferon-beta $1 \mathrm{~b}$, glatiramer acetate, pegylated interferon-beta la, alemtuzumab, mitoxantrone, and natalizumab.

\section{Trends in Oral DMD Use}

We evaluated changes over time in the proportion of subjects initiating oral DMDs and, separately, the proportion of subjects switching from an injectable DMD to another DMD that was accounted for by oral DMDs. Calendar dates of DMD initiation or switching were identified using prescription dispensing records. Based on these dates, semiannual proportions of DMD initiations and switches were plotted between July 2010 (to coincide with the first 6-month period covering the approval of fingolimod, the first oral agent, in September 2010) and December 2014

\section{Predictors of Oral DMD Use}

To evaluate predictors of treatment with oral DMDs, we matched oral DMD initiators with injectable DMD initiators and oral DMD switchers with patients switching to a second injectable DMD. Patients were matched on dates ( \pm 90 days) of their index visits and DMD initiation or switch. This risk-set sampling approach created matched sets of oral and injectable DMD initiators (or switchers), ${ }^{11}$ who were dispensed prescriptions at similar time points, and allowed for the evaluation of predictors of interest while efficiently controlling for the confounding effect of changing prescriber preferences with calendar time. For the initiation sample, up to 10 injectable DMD initiators were matched to each oral DMD initiator. Because of the smaller pool of eligible patients switching from one injectable to a second injectable agent, we matched oral and injectable switchers in a 1:1 ratio in the switching sample.

We then assessed the following sets of variables as potential factors associated with initiating or switching to oral DMDs:

1. Patient demographics, including patient gender, age, and geographic region of residence.

2. MS symptomatic treatment. We considered a broad range of medications commonly used to treat MS-related symptoms as indicators for patient quality of life because these symptoms can be highly burdensome for patients. ${ }^{12}$ We identified treatment for walking difficulties (dalfampridine; bladder problems (desmopressin, botulinum toxin, oxybutynin, and tolterodine); fatigue (amantadine, modafinil, armodafinil, and methylphenidate); erectile dysfunction (sildenafil citrate, tadalafil, and vardenafil); and acial neuralgia or spasticity (carbamazepine, gabapentin, 
lamotrigine, phenytoin, pregabalin, baclofen, diazepam, clonazepam, dantrolene, and tizanidine). We also identified use of intravenous steroids or high-dose oral steroids (defined as equivalent to more than $1,000 \mathrm{mg}$ of prednisone) as markers for acute MS relapse. ${ }^{13}$ Use of all these medications was measured in the 6-month period before the date of first DMD initiation or switch after the index date.

3. Comorbid diagnosis. We identified clinical conditions potentially associated with treatment selection, including seizures; depression; gastrointestinal symptoms (nausea or vomiting, abdominal pain, and diarrhea); hematologic conditions (thrombocytopenia, neutropenia, leukocytopenia, and lymphocytopenia); liver diseases (hepatitis, chronic liver disease, or cirrhosis); cardiovascular diseases (ischemic heart diseases, cerebrovascular events, or chronic heart failure); diabetes mellitus; and hypertension. We used diagnosis codes recorded during a medical visit any time before the DMD initiation or switch of interest to identify these conditions. We also identified diagnosis of serious bacterial infections (meningitis, encephalitis, cellulitis, endocarditis, pneumonia, pyelonephritis, osteomyelitis, and bacteremia); opportunistic infections (tuberculosis, systemic candidiasis, cryptococcosis, and aspergillosis); or viral infections (herpes zoster and varicella zoster) in the 6 months before the DMD initiation or switch date.

4. Health care use factors. We also identified any consultations with a neurologist or occupational or physical therapists and any hospitalization and emergency department visit as markers for general patient health and contact with the health care system. These visits were assessed in the 6-month period before the DMD initiation or switch of interest.

\section{Statistical Analysis}

Cochran-Armitage trend tests were conducted to evaluate statistical significance in utilization trends for DMD initiation and switching over time. Conditional logistic regression models were used to account for the matched sampling design in the analysis of predictors among initiators and switchers separately. All 4 sets of predictors previously described were included in the multivariable models to estimate odds ratios (OR) and 95\% confidence intervals (CI) for each predictor while controlling for all others. Tests for statistical significance were conducted for an alpha level of 0.05. All analyses were conducted using SAS version 9.4 (SAS Institute, Cary, NC).

\section{Results}

We identified 17,748 patients with a recorded MS diagnosis, of whom 7,576 DMD initiators and 1,342 DMD switchers were included in our study cohort. A majority of patients in both cohorts were aged $18-49$ years; $75 \%$ were female; and approximately $70 \%$ lived in the South or Northeast (Table 1). The switching population had a higher prevalence of MS symptomatic treatment use, comorbid diagnosis, and health care use compared with the initiation population (Table 1). The oral DMDs accounted for 477 (6\%) DMD initiations and 525 (39\%) DMD switches in our cohort. Figure 1 summarizes the number of patients included at various stages of this study.

\section{Trends in DMD Initiation and Switching}

Figure 2 presents trends in initiation and switching patterns of DMDs. Among patients newly starting DMDs, we observed a substantial increase in oral DMD use from the second half of 2012 to the second half of 2013, reaching 24\% of total DMD initiations before declining to 15\% of DMD initiations in the second half of $2014(P<0.0001)$. DMF accounted for the majority of oral DMD initiations over the study period (270 of 477 , or $57 \%, P<0.0001$ ). Fingolimod showed a modest uptake after its introduction (7\% of total DMD initiations in the second half of 2010), but its relative use declined substantially after DMF became available, accounting for only $2 \%$ in the second half of $2014(P=0.78)$. Teriflunamide was infrequently used in this population throughout the study period $(P=0.45)$.

Among DMD switchers, the proportion of patients switching to an oral agent increased from 6\% in the second half of 2010 to $79 \%$ in the second half of $2014(P<0.0001)$. Among the specific agents, DMF was again the most commonly dispensed DMD in this population, accounting for approximately $60 \%$ of switches since its introduction $(P<0.0001)$. Fingolimod accounted for $41 \%$ of switches in the first half of 2011 but declined substantially to $23 \%$ of switches in the second half of $2014(P=0.06)$. Teriflunamide was the least preferred agent, only dispensed in $4 \%$ of switches in the second half of $2014(P=0.07)$.

\section{Predictors of Oral DMD Use}

We matched 422 oral DMD initiators to 3,176 injectable DMD initiators, using a variable ratio matching strategy, and 408 oral DMD switchers were matched to 408 injectable DMD switchers. Characteristics of the cohort are presented in Table 1. Among DMD initiators, patients starting oral DMDs were more likely to use MS symptomatic treatments, including dalfampridine, facial neuralgia or spasticity treatments, and steroids, and had a higher prevalence of depression and gastrointestinal symptoms (nausea or vomiting, abdominal pain, and diarrhea), compared with injectable DMD initiators. In the switching sample, oral and injectable DMD users were similar for most characteristics.

The result of the multivariable regression models accounting for all potential predictors simultaneously are presented in Figure 3. In the initiation sample, older ( $\geq 65$ years) as compared with younger (18-49 years) age was associated with a lower odds of oral DMD use (OR $=0.42,95 \% \mathrm{CI}=0.18$ 0.97). Use of dalfampridine ( $\mathrm{OR}=2.24,95 \% \mathrm{CI}=1.34-3.75)$, a neurologist consultation $(\mathrm{OR}=1.60,95 \% \mathrm{CI}=1.20-2.15)$, or emergency department visit $(\mathrm{OR}=1.43,95 \% \mathrm{CI}=1.01-2.01)$ had statistically significant association with higher odds of 
TABLE 1 Characteristics of MS Patients Included in the Study Cohort

\begin{tabular}{|c|c|c|c|c|c|c|}
\hline \multirow[b]{2}{*}{ Variable, n (\%) } & \multirow{2}{*}{$\begin{array}{c}\text { Initiation } \\
\text { Population for } \\
\text { Time-Trend } \\
\text { Analysis } \\
\\
\text { Total } \\
(\mathrm{N}=7,576)\end{array}$} & \multicolumn{2}{|c|}{$\begin{array}{l}\text { Calendar Time-Matched Sample } \\
\text { for Predictors Analysis }\end{array}$} & \multirow{2}{*}{$\begin{array}{c}\text { Switching } \\
\text { Population for } \\
\text { Time-Trend } \\
\text { Analysis } \\
\\
\text { Total } \\
(\mathrm{N}=1,342)\end{array}$} & \multicolumn{2}{|c|}{$\begin{array}{c}\text { Calendar Time-Matched Sample } \\
\text { for Predictors Analysis }\end{array}$} \\
\hline & & $\begin{array}{l}\text { Oral Initiators } \\
\qquad(\mathrm{n}=422)\end{array}$ & $\begin{array}{l}\text { Injectable } \\
\text { Initiators } \\
(\mathrm{n}=3,176)\end{array}$ & & $\begin{array}{l}\text { Oral Switchers } \\
\qquad(\mathrm{n}=408)\end{array}$ & $\begin{array}{c}\text { Injectable } \\
\text { Switchers } \\
(\mathrm{n}=408)\end{array}$ \\
\hline \multicolumn{7}{|l|}{ Demographics } \\
\hline \multicolumn{7}{|l|}{ Age, years } \\
\hline $18-49$ & $4,698(62.0)$ & $272(64.5)$ & $2,025(63.8)$ & $862(64.2)$ & $267(65.4)$ & $257(63.0)$ \\
\hline $50-64$ & $2,730(36.0)$ & $141(33.4)$ & $1,079(34.0)$ & $451(33.6)$ & $126(30.9)$ & $143(35.0)$ \\
\hline$\geq 65$ & $148 \quad(2.0)$ & $9 \quad(2.1)$ & $72 \quad(2.3)$ & $29 \quad(2.2)$ & $15 \quad(3.7)$ & $8 \quad(2.0)$ \\
\hline Females & $5,681 \quad(75.1)$ & $320(75.8)$ & $2,320(73.3)$ & $1,006(75.1)$ & $300(73.7)$ & $287(70.9)$ \\
\hline \multicolumn{7}{|l|}{ Region $^{\mathrm{a}}$} \\
\hline Midwest & $822(10.9)$ & $27 \quad(6.4)$ & $242 \quad(7.6)$ & $122 \quad(9.1)$ & $36 \quad(8.8)$ & $25 \quad(6.1)$ \\
\hline Northeast & 2,567 (33.9) & $124(29.4)$ & $1,373(43.2)$ & $454(33.8)$ & $118(28.9)$ & $162(39.7)$ \\
\hline South & $2,701 \quad(35.7)$ & $182(43.1)$ & 981(30.9) & $494(36.8)$ & $165(40.4)$ & $140(34.3)$ \\
\hline West & $1,336 \quad(17.6)$ & $82(19.4)$ & $513(16.2)$ & $243(18.1)$ & $84(20.6)$ & $78(19.1)$ \\
\hline \multicolumn{7}{|l|}{ Treatment for MS-associated symptoms ${ }^{b}$} \\
\hline Dalfampridine & $203 \quad(2.7)$ & $31 \quad(7.3)$ & $121 \quad(3.8)$ & $103 \quad(7.7)$ & $(9.8)$ & $52(12.7)$ \\
\hline Continence treatment & $364 \quad(4.8)$ & $16 \quad(3.8)$ & $130 \quad(4.1)$ & $81 \quad(6.0)$ & $24 \quad(5.9)$ & $18 \quad(4.4)$ \\
\hline Fatigue treatment & $921(12.2)$ & $46(10.9)$ & $339(10.7)$ & $181(13.5)$ & $61(15)$ & $36 \quad(8.8)$ \\
\hline Erectile dysfunction treatment & $75 \quad(1.0)$ & $6 \quad(1.4)$ & $19 \quad(0.6)$ & $25 \quad(1.9)$ & $8 \quad(2.0)$ & $6 \quad(1.5)$ \\
\hline Neuralgia or muscle spasm treatment & $2,369 \quad(31.3)$ & $155(36.7)$ & $969(30.5)$ & $528(39.3)$ & $155(38)$ & $164(40.2)$ \\
\hline Injectable steroids & $833(11.0)$ & $350(11.0)$ & $53(12.6)$ & $197(14.8)$ & $59(14.5)$ & $80(19.6)$ \\
\hline Oral high-dose steroids ${ }^{\mathrm{c}}$ & $192(2.5)$ & $21 \quad(5.0)$ & $98 \quad(3.1)$ & $56 \quad(4.2)$ & $19 \quad(4.7)$ & $24 \quad(5.9)$ \\
\hline \multicolumn{7}{|l|}{ Comorbid diagnosis and health care use } \\
\hline Seizures & $209 \quad(2.8)$ & $16 \quad(3.8)$ & $125 \quad(3.9)$ & $53 \quad(4.0)$ & $22 \quad(5.4)$ & $22 \quad(5.4)$ \\
\hline Depression & $1,242(16.4)$ & $112(26.5)$ & $544(17.1)$ & $316(23.6)$ & $99(24.3)$ & $102(25.0)$ \\
\hline Gastrointestinal symptoms & $963(12.7)$ & $97(23.0)$ & $531(16.7)$ & $282(21)$ & $102(25.0)$ & $95(23.3)$ \\
\hline Hematological conditions & $97 \quad(1.3)$ & $10 \quad(2.4)$ & $51 \quad(1.6)$ & $28 \quad(2.1)$ & $7 \quad(1.7)$ & $14 \quad(3.4)$ \\
\hline Liver diseases & $62 \quad(0.8)$ & $7 \quad(1.7)$ & $40 \quad(1.3)$ & $27 \quad(2.0)$ & $11 \quad(2.7)$ & $11 \quad(2.7)$ \\
\hline Hypertension & $1,524(20.1)$ & $102(24.2)$ & $692(21.8)$ & $327(24.4)$ & $101(24.8)$ & $92(22.5)$ \\
\hline Diabetes & $414 \quad(5.5)$ & $29 \quad(6.9)$ & $200 \quad(6.3)$ & $89 \quad(6.6)$ & $33 \quad(8.1)$ & $25 \quad(6.1)$ \\
\hline Cardiovascular diseases $^{\mathrm{d}}$ & $373 \quad(4.9)$ & $30 \quad(7.1)$ & $200 \quad(6.3)$ & $(7.3)$ & $(8.1)$ & $(6.9)$ \\
\hline Infections $^{\mathrm{e}}$ & $370 \quad(4.9)$ & $20 \quad(4.7)$ & $141 \quad(4.4)$ & $(5.2)$ & $(6.1)$ & $(4.4)$ \\
\hline Any hospitalization in previous 6 months & $463 \quad(6.1)$ & $29 \quad(6.9)$ & $228 \quad(7.2)$ & $87 \quad(6.5)$ & $18 \quad(4.4)$ & $26 \quad(6.4)$ \\
\hline $\begin{array}{l}\text { Any neurologist consultation in previous } \\
6 \text { months }\end{array}$ & $6,069(80.1)$ & $325(77.0)$ & $2,365(74.5)$ & $1,126(83.9)$ & $323(79.2)$ & $344(84.3)$ \\
\hline $\begin{array}{l}\text { Any occupational or physical therapy } \\
\text { visits in previous } 6 \text { months }\end{array}$ & $292 \quad(3.9)$ & $14 \quad(3.3)$ & $107 \quad(3.4)$ & $73 \quad(5.4)$ & $19 \quad(4.7)$ & $33(8.1)$ \\
\hline $\begin{array}{l}\text { Any emergency department visits in } \\
\text { previous } 6 \text { months }\end{array}$ & $1,218(16.1)$ & $82(19.4)$ & $520(16.4)$ & $212(15.8)$ & $59(14.5)$ & $57(14.0)$ \\
\hline
\end{tabular}

a Region missing for approximately $2 \%$ of patients.

bIncontinence treatment included desmopressin, botulinum toxin, oxybutynin, and tolterodine; fatigue treatment included amantadine, modafinil, armodafinil, and methylphenidate; erectile dysfunction treatment included sildenafil citrate, tadalafil, and vardenafil; and neuralgia or muscle spasm treatment included carbamazepine, gabapentin, lamotrigine, phenytoin, pregabalin, baclofen, diazepam, clonazepam, dantrolene, and tizanidine.

cOral high-dose steroids were defined by a cumulative dose of oral steroid equivalent to more than 1,000 $\mathrm{mg}$ of prednisone.

${ }^{d}$ Cardiovascular diseases included ischemic heart diseases, cerebrovascular events, or chronic heart failure.

eInfections included serious bacterial infections (meningitis, encephalitis, cellulitis, endocarditis, pneumonia, pyelonephritis, osteomyelitis, and bacteremia) and opportunistic infections (tuberculosis, systemic candidiasis, cryptococcosis, and aspergillosis) or herpes zoster in the 6 months before DMD initiation or switch.

$\mathrm{DMD}=$ disease- modifying drug; MS = multiple sclerosis

oral DMD initiation. History of depression was noted to be a potential predictor of oral DMD initiation; however, the estimate for this predictor did not reach statistical significance
$(\mathrm{OR}=1.35,95 \% \mathrm{CI}=0.99-1.84)$. In the switching sample, the only significant predictor of oral DMD use was Northeast versus South regions $(\mathrm{OR}=0.66,95 \% \mathrm{CI}=0.46-0.96)$. 


\section{FIGURE 1 Sample Derivation Flowchart}

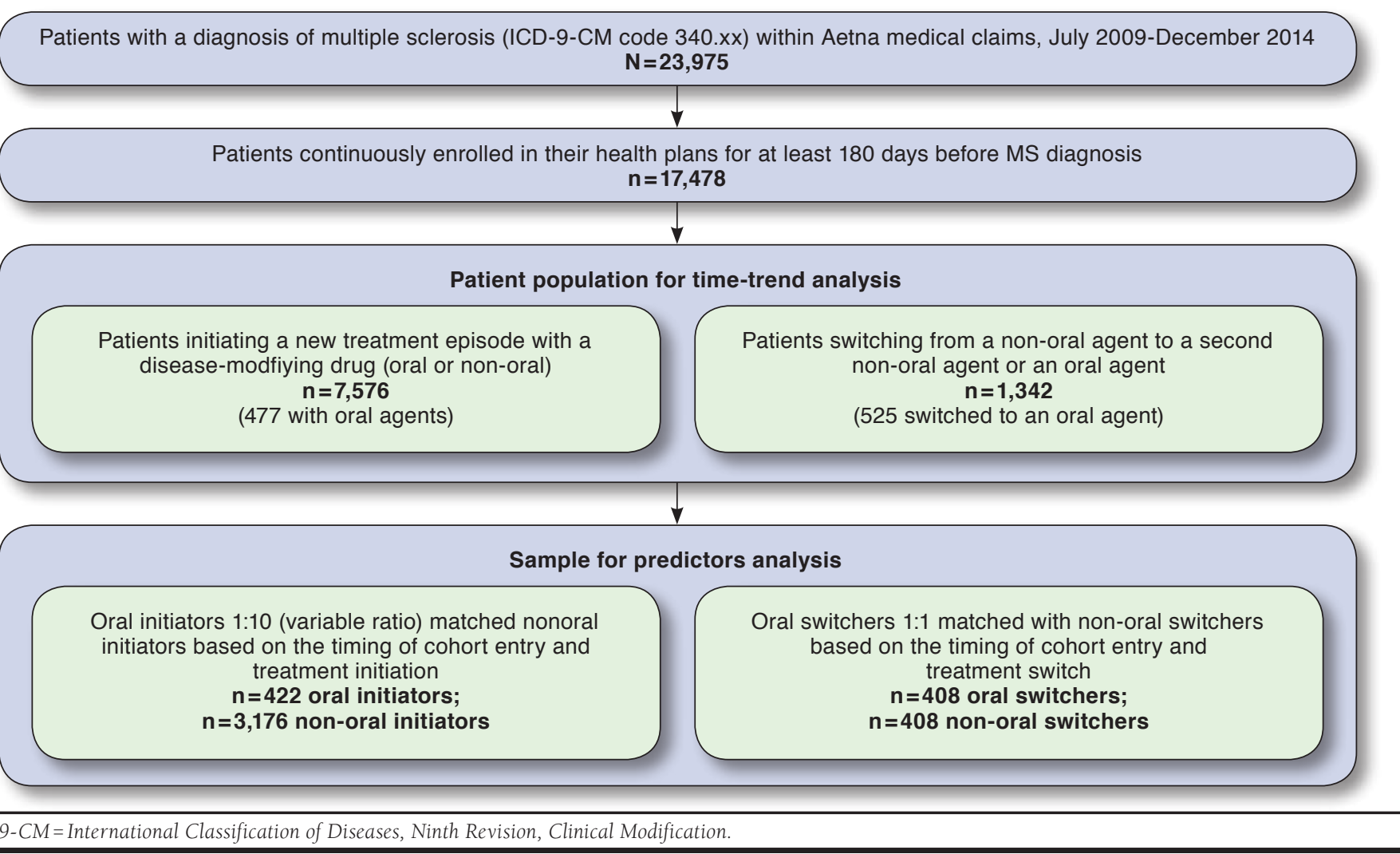

\section{Discussion}

Our study of real-world and contemporary DMD treatment patterns for patients with MS demonstrates the widespread adoption of oral DMDs, especially as second-line agents. These drugs now account for 15\% of DMD initiations and $80 \%$ of DMD switches. While some groups appear to be more likely to initiate MS treatment with an oral agent, including patients who are younger and with access to neurologists, we found few predictors with clinical significance of oral DMD initiation and none for switching to oral DMDs. This finding suggests a strong role of additional predictors not captured in this study, such as patient or provider preferences, as well as formulary status and insurance coverage of treatment options in the decision to initiate or switch to an oral DMD for MS.

While the oral agents have accumulated a sizable proportion of the DMD market share, use of some of these agents as first-line treatment options for MS is uncommon. For example, fingolimod, the first available oral DMD, remained infrequent as the first-line treatment for MS, accounting for only 3.6\% of new DMD initiations during the study period, which is in line with recommendations that suggest reserving fingolimod therapy for patients who do not benefit from or cannot tolerate the platform injectable agents. ${ }^{14}$ The use of teriflunamide also remained infrequent in the study population. However, DMF became a dominant treatment option soon after its introduction in March 2013, accounting for nearly 20\% of new DMD initiations by December 2013 and resulting in corresponding reductions in the use of other oral DMDs and injectable agents. Our data suggest that while the oral agents account for nearly 1 in 7 first-line initiations in MS patients, these agents have not completely replaced the platform injectable agents as some experts had anticipated at the time of their introduction. ${ }^{15}$ In fact, we observed a decreasing trend in DMF initiations and switches in 2014, which appears to coincide with rising concerns over the risk of progressive multifocal leukoencephalopathy after DMF treatment. ${ }^{6,7}$

Among patients initiating new DMDs as first-line agents, we noted that older age was negatively associated with oral DMD initiation, which may reflect concerns about cardiovascular adverse events and varicella-zoster virus reactivation associated with fingolimod, ${ }^{14,16}$ the risks of which, at baseline, are much higher among older individuals. ${ }^{5}$ The recent use of dalfampridine, a treatment for MS-associated walking difficulties, and a recent emergency department visit predicted the use of 


\section{FIGURE 2 Time Trends in Initiation and Switching Patterns of DMDs in Patients with MS}

\section{A. Initiations}

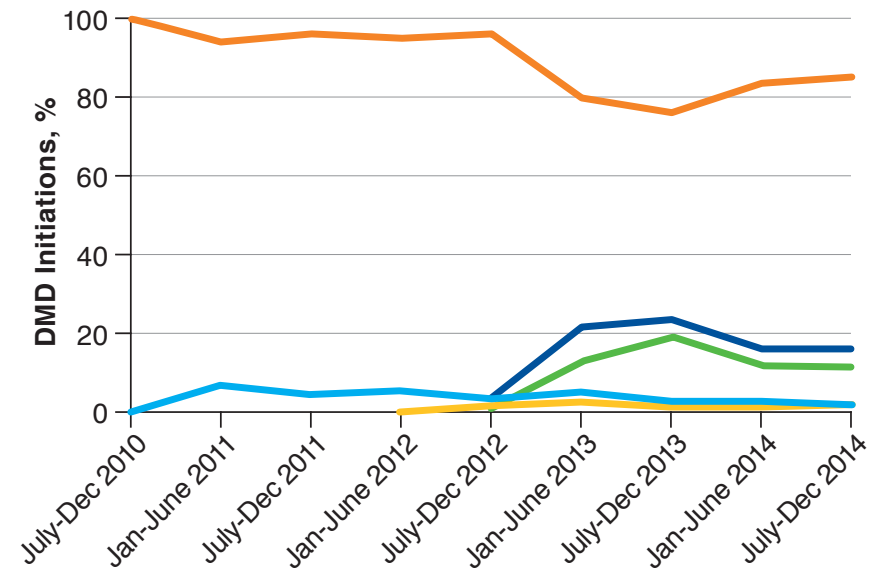

\section{B. Switching Patterns}

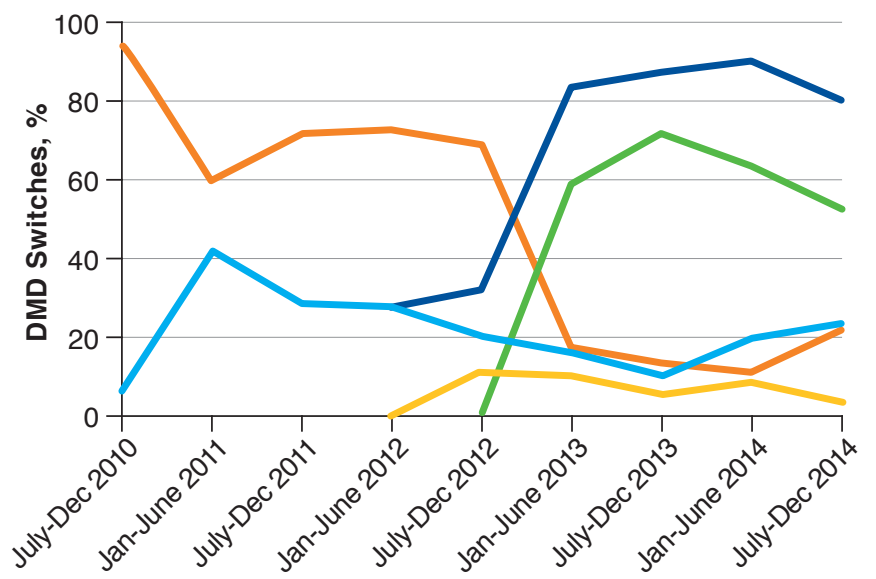

Fingolimod $\longrightarrow$ Dimethyl fumarate

- Teriflunamide

Injectable DMDs Oral DMDs

DMD = disease-modifying drug; MS = multiple sclerosis

oral DMDs, suggesting that patients starting treatment with an oral agent may have had more severe disease. A diagnosis of depression was associated with oral DMD initiation as firstline agents, albeit a statistically nonsignificant association, which potentially indicates channeling of MS patients with depression away from interferon therapy because of concerns about interferon-associated worsening of depression. ${ }^{17}$ We also found a strong association between a neurologist consultation and initiation of oral DMDs as first-line agents, indicating that access to specialists, who may be more familiar and comfortable with using newer agents, is an important determinant of the first DMD in patients with MS.

In contrast to the treatment patterns among initiators, we observed that oral agents accounted for nearly 8 out of 10 treatment switches by the end of 2014, with DMF again being the dominant choice. This finding suggests that providers consider the potential adverse events as a barrier to more widespread use of oral agents as first-line options and may only prescribe these agents in situations of inadequate response, tolerability, or strong patient and physician preferences after trying treatment with the platform injectable agents. Consistent with this, among MS patients using injectable therapy, few factors predicted switching to oral DMDs versus another injectable agent, suggesting that their use may be driven in part by preferences. For example, the absence of an association in the data between switching to an oral agent and factors indicating MS relapse, including high-dose steroid use and hospitalization, may indicate patient-specific concerns about tolerability or route of administration rather than treatment selection based on treatment failure or relapse.
That said, early reactivation of disease has been documented in previous studies after treatment switches to oral DMDs mainly for reasons unrelated to efficacy. ${ }^{18,19}$

Although, a recent study observed no increased risk of early relapse in stable patients switching to fingolimod from platform injectable agents, ${ }^{20}$ our data demonstrating the high frequency of switching to oral DMDs highlight the need for continued research on optimal switching approaches to ensure maximum benefits in patients who are stable on their initial injectable treatments but switch to an oral agent due to tolerability concerns or preference for an oral route.

\section{Strengths and Limitations}

Our study has several unique strengths. Owing to the availability of comprehensive data on patient prescription drug use, as well as medical visits, we were able to describe correlates of oral DMD use in our sample after carefully accounting for changing prescriber practices through a calendar time-matched design. In order to appropriately characterize factors influencing oral DMD treatment at different stages of their disease, we separately evaluated predictors in early MS patients initiating DMDs and late-stage MS patients switching to another DMD.

There are also several limitations to this study that merit discussion. First, we did not have data on factors indicating patient clinical disease activity or disability status, which may be strong predictors of treatment choice. To address this limitation, we used several proxy variables (e.g., treatment with agents commonly used for MS symptoms) and health care use factors (e.g., hospitalization and emergency department visits). 
FIGURE 3 Factors Associated with Initiation of and Switching to Oral DMDs in Patients with MS

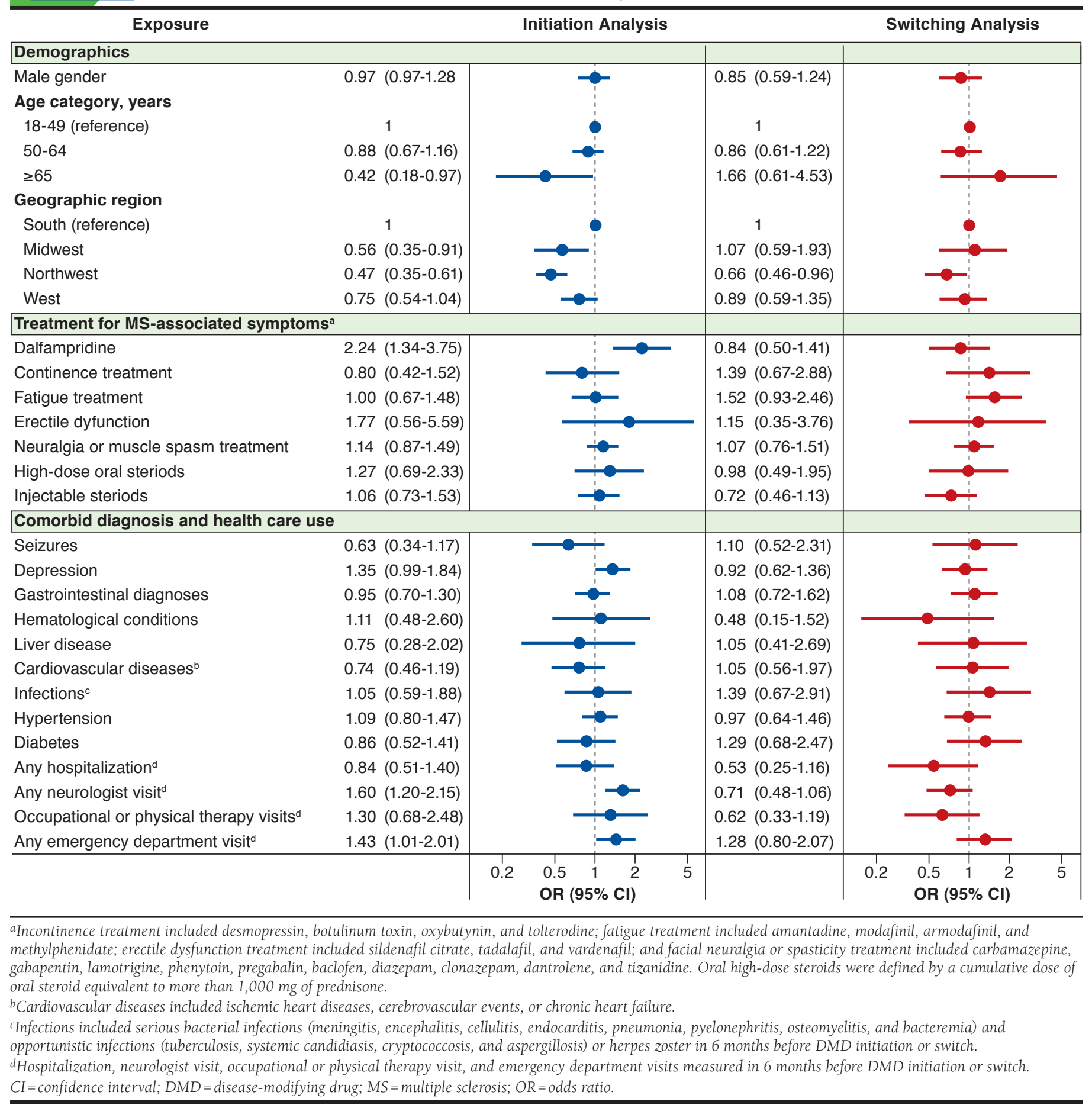

However, other data sources that may contain additional information regarding patient disease severity, such as electronic medical records, could be used to augment insurance claims to identify relevant predictors.
Second, we were not able to validate the diagnosis of MS identified in administrative claims against medical records. However, a previous validation study noted high positive predicted value of our approach, which included patients with MS 
diagnosis and at least 1 filled DMD prescription in administrative claims. ${ }^{10}$

Third, we did not have data on some potentially important patient factors, such as race, income levels, educational attainment, which limited our ability to explore disparities in use of oral DMDs by these factors. Further, we did not collect data on coverage factors such as formulary status of individual agents, which may be important factors in determining treatment decisions in MS patients. In a recent study, monthly out-of-pocket cost responsibility was rated as the most influential attribute by patients in determining their preference for DMDs. ${ }^{21}$ Therefore, unavailability of this information is an important limitation of this study, and future research should elaborate on the influence of these factors on treatment choice.

Finally, our results are only directly applicable to patients with employer-sponsored private health insurance coverage and may have limited generalizability to patients with public insurance coverage or no insurance. Further, we could not differentiate whether patients who initiated therapy on oral DMDs explicitly requested such treatment or whether provider preferences drove these patterns. Future survey research that explores the source of these patterns would be valuable.

\section{Conclusions}

Our study demonstrated widespread adoption of oral DMDs in routine clinical practice as alternatives to injectable DMDs for MS, especially for second-line treatment. The rapid increase in use of oral DMDs observed in our study highlights the need for future research examining their comparative effectiveness and safety.

\section{Authors}

RISHI J. DESAI, MS, PhD; MUFADDAL MAHESRI, MD, PhD; JOSHUA J. GAGNE, PharmD, ScD; ANGELA TONG, MS; and NITEESH K. CHOUDHRY, MD, PhD, Division of Pharmacoepidemiology and Pharmacoeconomics, Department of Medicine, Brigham and Women's Hospital/Harvard Medical School, Boston, Massachusetts. TANUJA CHITNIS, MD, Department of Neurology, and SARAH MINDEN, MD, Department of Psychiatry, Brigham and Women's Hospital/Harvard Medical School, Boston, Massachusetts. EIMIR HURLEY, MS, Centre for Health Policy and Management, Trinity College, Dublin, Ireland, and CLAIRE M. SPETTELL, PhD, Aetna, Hartford, Connecticut. OLGA S. MATLIN, $\mathrm{PhD}$, and WILLIAM H. SHRANK, MD, MS, CVS Caremark, Woonsocket, Rhode Island.

AUTHOR CORRESPONDENCE: Rishi J. Desai, MS, PhD, Division of Pharmacoepidemiology \& Pharmacoeconomics, Brigham and Women's Hospital, 1 Brigham Cir., Ste. 3030, Boston, MA 02120. Tel.: 617.278.0932; E-mail: rdesai@bwh.harvard.edu.

\section{DISCLOSURES}

This study was funded by CVS Caremark through an unrestricted research grant to Brigham and Women's Hospital. Shrank and Matlin were employees of, and shareholders in, CVS Health at the time of the study; they report no financial interests in products or services that are related to the subject of this study. Spettell is an employee of, and shareholder in, Aetna. Chitnis serves on clinical trial advisory boards for Novartis and Genzyme-Sanofi; has consulted for Bayer, Biogen Idec, Celgene, Novartis, Merck-Serono, and Genentech-Roche; and has received research support from NIH, National Multiple Sclerosis Society, Peabody Foundation, Consortium for MS Centers, Guthy Jackson Charitable Foundation, EMD-Serono, Novartis Biogen, and Verily. Desai reports receiving a research grant from Merck for unrelated work. Gagne is principal investigator of a research grant from Novartis Pharmaceuticals Corporation to the Brigham and Women's Hospital and has received grant support from Eli Lilly, all for unrelated work. He is also a consultant to Aetion and Optum. Minden reports grants from Biogen and other fees from Genentech, EMD Serano, Avanir, and Novartis, unrelated to this study. The other authors have no conflicts to report.

This study was presented as a poster at the International Society for Pharmacoepidemiology 32nd Annual Meeting; August 25-28, 2016; Dublin, Ireland.

\section{REFERENCES}

1. Freedman MS, Hughes B, Mikol DD, et al. Efficacy of disease-modifying therapies in relapsing remitting multiple sclerosis: a systematic comparison. Eur Neurol. 2008;60(1):1-11.

2. Klauer T, Zettl UK. Compliance, adherence, and the treatment of multiple sclerosis. J Neurol. 2008;255(Suppl 6):87-92

3. Higuera L, Carlin CS, Anderson S. Adherence to disease-modifying therapies for multiple sclerosis. J Manag Care Spec Pharm. 2016;22(12):1394-401. Available at: https://www.jmcp.org/doi/10.18553/jmcp.2016.22.12.1394

4. Tolley K, Hutchinson M, You X, et al. A network meta-analysis of efficacy and evaluation of safety of subcutaneous pegylated interferon beta-la versus other injectable therapies for the treatment of relapsing-remitting multiple sclerosis. PloS One. 2015;10(6):e0127960.

5. Arvin AM, Wolinsky JS, Kappos L, et al. Varicella-zoster virus infections in patients treated with fingolimod: risk assessment and consensus recommendations for management. JAMA Neurology. 2015;72(1):31-39.

6. van Oosten BW, Killestein J, Barkhof F, Polman CH, Wattjes MP. PML in a patient treated with dimethyl fumarate from a compounding pharmacy. New Engl J Med. 2013;368(17):1658-59.

7. Ermis U, Weis J, Schulz JB. PML in a patient treated with fumaric acid. N Engl J Med. 2013;368(17):1657-58.

8. Williamson EM, Berger JR. Infection risk in patients on multiple sclerosis therapeutics. CNS Drugs. 2015;29(3):229-44.

9. English C, Aloi JJ. New FDA-approved disease-modifying therapies for multiple sclerosis. Clin Ther. 2015;37(4):691-715.

10. Culpepper WJ 2nd, Ehrmantraut M, Wallin MT, Flannery K, Bradham DD. Veterans Health Administration multiple sclerosis surveillance registry: the problem of case-finding from administrative databases. J Rehabil Res Dev. 2006;43(1):17-24

11. Rothman KJ, Greenland S, Lash TL. Modern Epidemiology. Philadelphia, PA: Lippincott Williams \& Wilkins; 2008.

12. Thompson AJ, Toosy AT, Ciccarelli O. Pharmacological management of symptoms in multiple sclerosis: current approaches and future directions. Lancet Neurol. 2010;9(12):1182-99.

13. Chastek BJ, Oleen-Burkey M, Lopez-Bresnahan MV. Medical chart validation of an algorithm for identifying multiple sclerosis relapse in healthcare claims. J Med Econ. 2010;13(4):618-25.

14. Pelletier D, Hafler DA. Fingolimod for multiple sclerosis. New Engl J Med. 2012;366(4):339-47 
15. Sorensen PS. New management algorithms in multiple sclerosis. Curr Opin Neurol. 2014;27(3):246-59.

16. Rimland D, Moanna A. Increasing incidence of herpes zoster among veterans. Clin Infec Dis. 2010;50(7):1000-05.

17. Siegert RJ, Abernethy DA. Depression in multiple sclerosis: a review. J Neurol Neurosurg Psychiatry. 2005;76(4):469-75.

18. Cohen M, Maillart E, Tourbah A, et al. Switching from natalizumab to fingolimod in multiple sclerosis: a French prospective study. JAMA Neurol. 2014;71(4):436-41
19. Comi G, Gold R, Dahlke F, et al. Relapses in patients treated with fingolimod after previous exposure to natalizumab. Mult Scler. 2015;21(6):786-90.

20. Spelman T, Mekhael L, Burke T, et al. Risk of early relapse following the switch from injectables to oral agents for multiple sclerosis. Eur J Neurol. 2016;23(4):729-36.

21. Hincapie AL, Penm J, Burns CF. Factors associated with patient preferences for disease-modifying therapies in multiple sclerosis. J Manag Care Spec Pharm. 2017;23(8):822-30. Available at: https://www.jmcp.org/ doi/10.18553/jmcp.2017.23.8.822. 